\title{
Bildgebung und Verhaltenstherapie: Chancen und Grenzen
}

\author{
Kerstin Konrad ${ }^{\mathrm{a}} \quad$ Ulrike Ehlert $^{\mathrm{b}}$ \\ ${ }^{\text {a } L e h r-~ u n d ~ F o r s c h u n g s g e b i e t ~ K l i n i s c h e ~ N e u r o p s y c h o l o g i e ~ d e s ~ K i n d e s-~ u n d ~ J u g e n d a l t e r s, ~ K l i n i k ~ f u ̈ r ~ K i n d e r-~ u n d ~ J u g e n d p s y c h i a t r i e ~ u n d ~}$ \\ -psychotherapie, Universitätsklinikum Aachen, Deutschland

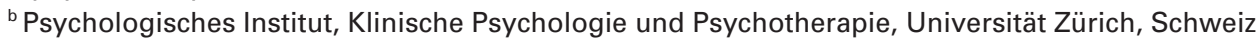

\begin{abstract}
«Die Biologie ist wahrscheinlich ein Reich der unbegrenzten Möglichkeiten, wir haben die überraschendsten Aufklärungen von ihr zu erwarten und können nicht erraten, welche Antworten sie auf die von uns an sie gestellten Fragen einige Jahrzehnte später geben würde. Vielleicht gerade solche, durch die unser ganzer künstlicher Bau von Hypothesen umgeblasen wird.» (S. Freud, 1920, Jenseits des Lustprinzips, S. 65)
\end{abstract}

Zwei große Entwicklungen prägen die moderne Neurowissenschaft. Zum einen werden immer präzisere Erkenntnisse über die molekularbiologischen Vorgänge im menschlichen Gehirn gewonnen. Zum anderen ermöglichen leistungsfähigere Bildgebungsverfahren es, Hirnfunktionen und mögliche Störungen dieser Funktionen besser zu verstehen.

Auch Psychotherapeuten werden sich zukünftig verstärkt mit der Wirkung ihrer Interventionen auf das Gehirn auseinandersetzen müssen, um die «Black Box» der neurobiologischen Veränderungen zu verstehen, die durch verschiedene Therapieansätze erzielt werden können. Dass diese Idee keineswegs neu ist, verdeutlicht das obige Zitat Sigmund Freuds aus dem Jahr 1920. Dennoch scheint uns Psychotherapeuten dieser Ansatz nach wie vor häufig unzureichend, und die Vorstellung, dass die Ursachen unseres Verhaltens in kausalen neuronalen Prozessen bestehen, zu reduktionistisch. Wie stark uns solche Fragen beschäftigen, verdeutlichen auch die derzeit kontrovers geführten Diskussionen zu kulturell geprägten Begriffen wie «Willensfreiheit» «Bewusstsein» oder «Verantwortung» zwischen Neurophilosophen und ihren Gegnern in den Medien [Churchland, 2002].

Prinzipiell machen Psychotherapieforschung auf der einen und Neurowissenschaften auf der anderen Seite Aussagen über einander überlappende Gegenstandsbereiche. Während viele psychologische Theorien eher auf eine Makro-Ebene abzielen, entwickeln die Neurowissenschaften vielmehr Mikrotheorien, die versuchen, Einzelphänomene mithilfe von verschiedenen Messmethoden zu erfassen und zu erklären. Unter günstigen Bedingungen könnte so längerfristig ein Brückenschlag zwischen den Ebenen gelingen, der einen komplexen Makro-Zusammenhang derart vereinfacht, dass eine erfolgreiche Reduktion durch eine mikrotheoretische Erklärung möglich wird [Pawelzik, 2007]. Komplexe Phänomene können so auf das Zusammenspiel einfacherer Elemente zurückgeführt werden, ohne dass die Makrophänomene damit entwertet werden. Dies ist ein großes Potenzial der Neurowissenschaften. Aber auch wenn wir besser verstehen, wie das Verhalten unseres Patienten, dem wir in der Psychotherapie gegenübersitzen, durch Gehirnprozesse bestimmt wird, hat dies keinen Einfluss darauf, wie wir ihm als Person begegnen und dass spezifische neurowissenschaftlich basierte Therapiemethoden scheitern werden, wenn es nicht gelingt, eine positive Beziehung zum Patienten aufzubauen oder seine Motivation und Kooperation zu gewinnen.

Was sind derzeit konkrete klinische Anwendungen für die funktionelle Bildgebung in der Klinischen Psychologie?

Bislang haben Bildgebungsmethoden vor allem einen wichtigen Beitrag zur Grundlagenforschung und zur Spezifikation neurobiologisch fundierter Störungsmodelle geleistet. Jetzt scheint die Methodik an der Schwelle zur klinischen-therapeutischen Nutzbarkeit zu stehen. In einer aktuellen Studie konnten Klöppel und Kollegen [2008] zeigen, dass mithilfe einer computerbasierten Auswertung von MRT-Bildern mithilfe von Lernmaschinen die diagnostische Klassifikation von Patienten mit und ohne Alzheimer-Erkrankung besser gelang als durch klinisch erfahrene Radiologen, insbesondere hinsichtlich der Differenzialdiagnostik verschiedener neurodegenerativer Erkrankungen. Solche diagnostischen Ansätze sind auch für eine Vielzahl von psychischen Störungen denkbar, aber bislang noch nicht realisiert. Für den klinisch-therapeutischen Bereich scheinen folgende Ansätze besonders vielversprechend [Linden, 2006]:

\section{KARGER}

Fax +497614520714

Information@Karger.de

www.karger.com (c) 2009 S. Karger GmbH, Freiburg

Accessible online at:

www.karger.com/ver

Klinik für Kinder- und Jugendpsychiatrie und -psychotherapie

Universitätsklinikum Aachen

Neuenhofer Weg 21, 52074 Aachen, Deutschland

Tel. +49 241 80-89262, Fax -82544, KKonrad@ukaachen.de 
(1) Therapieevaluation;

(2) differenzielle Indikationsstellung;

(3)Entwicklung neurowissenschaftlich motivierter Therapieverfahren, wie z.B. Neuropsychotherapie und Neurofeedbackverfahren;

(4) Identifizierung von Zielarealen für nichtinvasive (z.B. transkranielle Magnetstimulation) oder invasive (z.B. Tiefenhirnstimulation) neurophysiologische Behandlungsmethoden.

Aber können wir heute mithilfe von Bildgebungsmethoden tatsächlich den Behandlungserfolg messen? Was bedeutet es für den längerfristigen Verlauf, wenn Patienten nach einer erfolgreichen Therapie eine verminderte Aktivierung in einem bestimmten Hirnareal aufweisen? Können solche Veränderungen besser das längerfristige Outcome der Patienten vorhersagen als Veränderungen in störungsspezifischen Fragebögen oder klinischen Interviews? Und ist es derzeit schon möglich, anhand von Aktivierungsdaten eine differenzielle Indikation für die optimale Behandlungsmethode zu stellen?

Im vorliegenden Themenheft wollen wir versuchen, uns diesen Fragen zu stellen und den Brückenschlag zwischen
Neurowissenschaften und Verhaltenstherapie anhand ausgewählter psychischer Störungen exemplarisch zu veranschaulichen. Chancen und Grenzen der Bildgebungsmethoden für die Weiterentwicklung von Störungsmodellen und psychotherapeutischen Behandlungstechniken werden kritisch reflektiert. Keineswegs geht es hier um die Ablösung klassischer psychologischer Verfahren durch biologische, sondern vielmehr um eine Verfeinerung der Indikationsstellung und Anwendung therapeutischer Techniken durch neurobiologische Erkenntnisse.

\section{Literatur}

Churchland PS: Brain-Wise. Studies in Neurophilosophy. Cambridge, MA, MITPress, 2002.

Klöppel S, Stonnington CM, Barnes J, Chen F, Chu C, Good CD, Mader I, Mitch-

RS: Accuracy of dementia diagnosis: a direct comparison between radiologists and a computerized method. Brain 2008;131(Pt 11):2969-74.

Linden DEJ: Perspektiven der funktionellen Bildgebung in der Psychiatrie. Dtsch Ärztebl 2006;1003(38):2472-2478.

Pawelzik M: Neuropsychotherapie; in Gauggel S, Hermann M (Hrsg):. Handbuch der Neuro- und Biopsychologie. Göttingen, Hogrefe, 2007. 